\title{
Major Successes of Theory-and-Experiment-Combined Studies in Surface Chemistry and Heterogeneous Catalysis
}

\author{
Gabor A. Somorjai · Yimin Li
}

Published online: 4 February 2010

(c) The Author(s) 2010. This article is published with open access at Springerlink.com

\begin{abstract}
Experimental discoveries followed by theoretical interpretations that pave the way of further advances by experimentalists is a developing pattern in modern surface chemistry and catalysis. The revolution of modern surface science started with the development of surfacesensitive techniques such as LEED, XPS, AES, ISS and SIMS, in which the close collaboration between experimentalists and theorists led to the quantitative determination of surface structure and composition. The experimental discovery of the chemical activity of surface defects and the trends in the reactivity of transitional metals followed by the explanations from the theoretical studies led to the molecular level understanding of active sites in catalysis. The molecular level knowledge, in turn, provided a guide for experiments to search for new generation of catalysts. These and many other examples of successes in experiment-and-theory-combined studies demonstrate the importance of the collaboration between experimentalists and theorists in the development of modern surface science.
\end{abstract}

Keywords Theory success - Surface chemistry · Catalysis

G. A. Somorjai $(\bowtie) \cdot$ Y. Li

Department of Chemistry, University of California, Berkeley, CA 94720, USA

e-mail: Somorjai@cchem.berkeley.edu

G. A. Somorjai · Y. Li

Materials Science Division, Lawrence Berkeley National Laboratory, Berkeley, CA 94720, USA

\section{Introduction}

Both experiment and theory are indispensable in modern surface science. Modern surface science is all about the molecular level knowledge of physical, chemical, and biological processes occurring in the nanometer scale vicinity of surfaces or interfaces [1]. In order to acquire information at a resolution of a few nanometers, the first thing to do is developing surface-sensitive experimental techniques. However, this task is not only for experimental scientists because, virtually, the data analysis for every surface-sensitive technique, from low-energy electron diffraction crystallography [2,3] (LEED) to high resolution electron energy loss spectroscopy $[4,5]$ (HREELS), and from scanning tunneling microscopy [6-9] (STM) to sum frequency generation spectroscopy $[10,11]$ (SFG), relies on sophisticated theories. After new experimental techniques developed, new experimental data start to be accumulated. At certain point, theoretical models are called for again to reconcile the experimental data. A good model not only helps experimentalists to rationalize their results, but also makes predictions that challenge the capability of available experimental techniques in terms of the spatial, time and energy resolutions, and guide experimentalists to design new experiments.

Jens Nørskov is a theorist who has had a profound impact in the field of surface science and heterogeneous catalysis. His contributions to the theories of surface chemical bonding and catalytic reaction over the past 30 years lead to a giant leap forward in our molecular level understanding of surface chemistry and heterogeneous catalysis [12-21]. Of course, his achievement is a result of his exceptional expertise in theoretical chemistry, but, from the point of view of an experimentalist, the more important factors perhaps are his willingness to work closely with 
experimentalists and his ability to grasp the essence of experimental development [22-28].

In this paper, we will show, from the point of view of an experimentalist, how theory and experiment can play complementary roles in surface science by several examples including the development of LEED surface crystallography, determining the complex structure of surface oxide, the development of surface chemical bonding theory, and the development of theory for surface catalytic reactions and its application to rational design of catalyst. Nørskov's contributions to the theory of surface chemical bonding and catalytic reaction will be highlighted. We hope that our discussion will stimulate more collaboration between theorists and experimentalists in the field.

\section{The Development of Low Energy Electron Diffraction (LEED) Surface Crystallography}

The phenomenon of electron diffraction was first predicted by de Broglie [29] due to the quantum particle-wave duality in 1924, and was observed 3 years later by Davisson and Germer [30, 31] when a well-collimated beam of electrons was directed onto a crystallized nickel sample. It was soon realized that, in principle, the LEED pattern contains the structure information of the first few layers of atoms at the surface of materials.

However, it took almost 60 years after the Davisson and Germer experiment to develop LEED fully into a prime tool for quantitatively determining complex surface structures [3, 32, 33]. The major obstacles in this development resided in both experimental technique and theoretical interpretation of the experimental data. From the experimental aspect, it is crucial firstly to create clean surfaces and maintain the sample in this state within the duration of the LEED measurement; secondly, the inelastically scattered electrons, which plague the diffraction pattern formed by the elastically scattered electrons, must be filtered out in the experiment. In the early 1960s, the first problem was solved by the development of Ultra-High-Vacuum technology together with methods such as Auger electron spectroscopy (AES) for preparing clean surfaces and monitoring their cleanliness [34].

A clever design of the experimental setup shown in Fig. 1a further improved the detection of LEED pattern. The introduction of a fluorescent screen enabled the simultaneous monitoring of the diffracted electron beams in different directions and shortened the time duration of experiments. By applying appropriate voltage bias on the hemispherical concentric grids (Fig. 1b), the inelastically scattered electrons were filtered out and the elastically scattered electrons were accelerated onto the fluorescent screen to make the diffraction pattern more readily

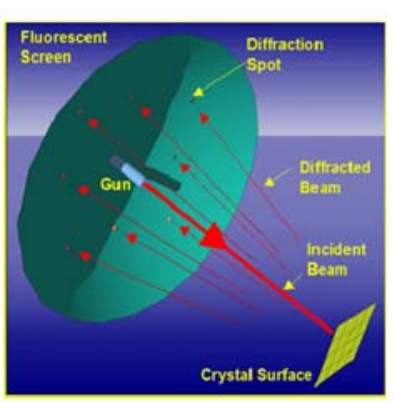

(a)

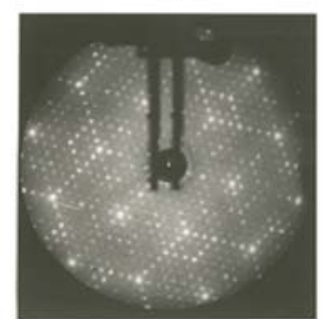

(c)

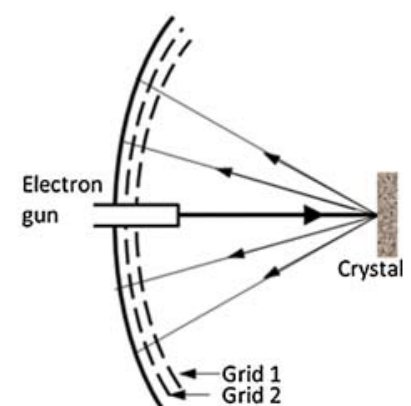

(b)
Fig. 1 a Schematic illustration of the LEED experimental setup. b A cross section view shows the hemispherical concentric grids used to filter out the inelastically scattered electrons. $\mathbf{c}$ The LEED pattern of the highly symmetric $(7 \times 7)$ reconstructed $\mathrm{Si}(111)$ surface

detectable. With these technical advances, the qualitative information such as the symmetry of the surface structure, the size and the rotational alignment of the adsorbate unit cell with respect to the substrate unit cell were readily obtained by analysis of the diffraction patterns from clean surfaces and surfaces with a given atomic adsorbate [2]. A spectacular example is the $(7 \times 7)$ reconstructed $\mathrm{Si}(111)$ surface shown in Fig. 1c. A total number of 49 surface atoms per unit cell are involved in the reconstruction of silicon surface atoms to generate this highly symmetric surface.

The quantitative information about exact atomic locations in surface layers can be extracted by theoretical analysis of the so-called $I-V$ curves, where the intensities of diffracted electron beams are recorded as a function of incident electron beam energy. In the 1960s, the theoretical method available for analyzing the $I-V$ curves was the kinematic theory derived from the $\mathrm{X}$-ray diffraction theory. Figure 2 a shows a successful analysis of $I-V$ curves for the (111) surface of solid xenon using the kinematic theory [35]. In this theory, it is assumed that every incident electron is scattered once by an atom in the surface layer before reaching the detector (Fig. 2b). This is true for the xenon case because of the uniquely short inelastic mean free path of low-energy electrons in solid xenon. For most materials, the multiple scattering processes as shown in Fig. $3 \mathrm{a}$ usually cannot be ignored. Figure $3 \mathrm{~b}$ demonstrates the necessity of the multiple scattering theory for fitting the $I-V$ from the $\mathrm{Cu}(001)$ surface [36]. 
Fig. 2 a The $I-V$ curve of the (111) surface of solid xenon. The solid curve is the experimental result. The dots is the result of a weak scattering theory. b The single electron scattering processes by atoms in one atomic layer (left) and in two atomic layers. $\mathbf{k}$ and $\mathbf{k}^{\prime}$ are the wave vectors of incident beam and diffracted beam, respectively. The interference between the diffracted beam is determined by $\Delta L$, the difference in their traveling distances
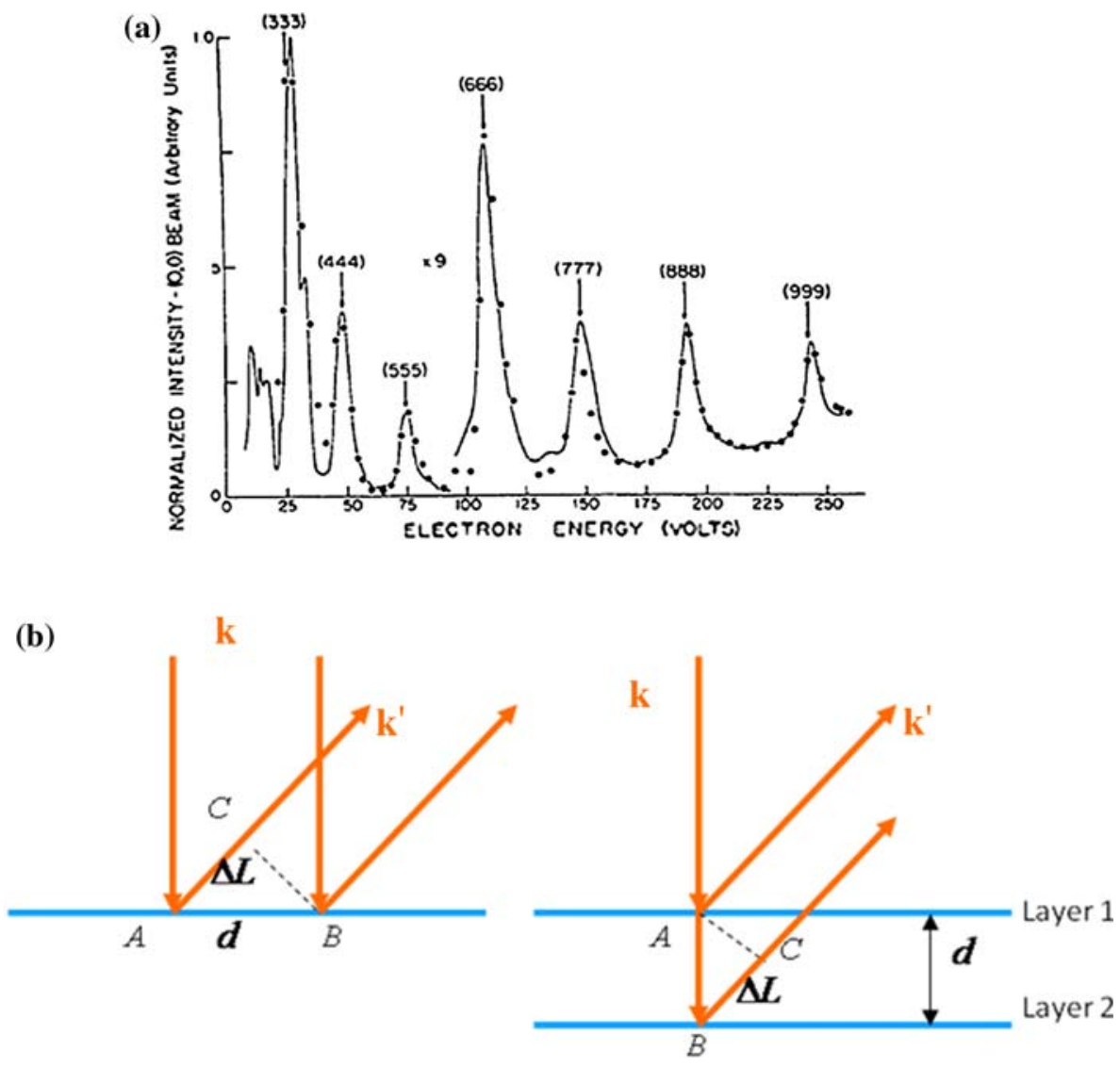
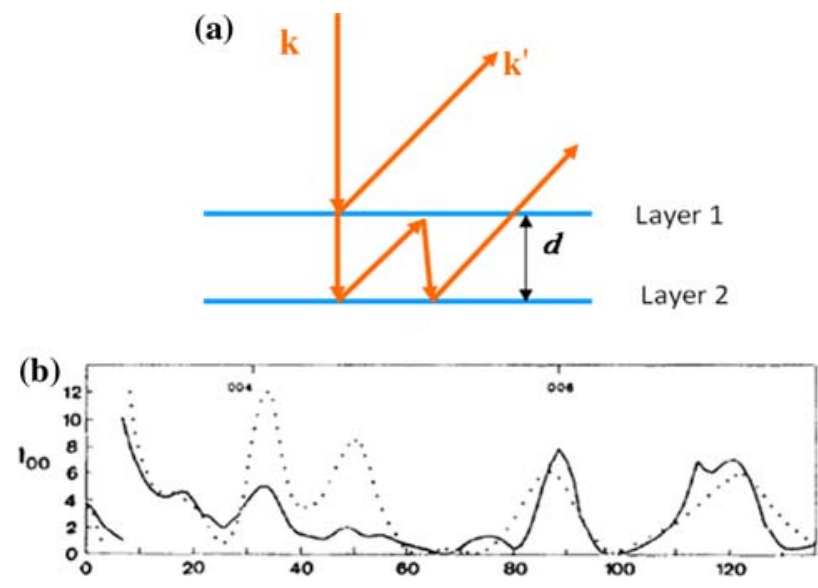

Fig. 3 a One possible multiple scattering processes of electrons by two atomic layers. b The $I-V$ curve of the $(0,0)$ beam diffracted from the $\mathrm{Cu}(011)$ surface. The solid lines show the results from a multiple scattering theory. Positions of peaks predicted by the single scattering theory are indicated at the top of the panel

Two computationally efficient methods developed by John B. Pendry [33] in the early 1970s revolutionized the calculation of the $I-V$ curves for comparison with experimental data. The first method was the so-called layerdoubling method which treats the multiple scattering in the surface layers explicitly. In this method, the surface is represented as a stack of identical $2 \mathrm{D}$ atomic planes. The basic idea is that once one has computed the transmission $\left(t_{1}\right)$ and the reflection $\left(r_{1}\right)$ coefficients for the single atomic layer (Fig. 4a), the reflection $\left(r_{2}\right)$ and the transmission $\left(t_{2}\right)$ coefficients of two atomic layer as shown in Fig. $4 \mathrm{~b}$ can be obtained as

$$
\begin{aligned}
r_{2} & =r_{1}+t_{1} r_{1} t_{1}+t_{1} r_{1} r_{1} r_{1} t_{1}+\cdots+t_{1} r_{1}^{2 n+1} t_{1}+\cdots \\
& =r_{1}+t_{1}\left(1-r_{1} r_{1}\right)^{-1} r_{1} t_{1}
\end{aligned}
$$

and

$$
\begin{aligned}
t_{2} & =t_{1} t_{1}+t_{1} r_{1} r_{1} t_{1}+t_{1} r_{1} r_{1} r_{1} r_{1} t+\cdots+t_{1} r_{1}^{2 n} t_{1}+\cdots \\
& =t_{1}\left(1-r_{1} r_{1}\right)^{-1} t_{1}
\end{aligned}
$$

Now the reflection $\left(r_{2^{i}}\right)$ and transmission $\left(t_{2^{i}}\right)$ coefficients of $2^{i}$ layers as shown in Fig. $4 \mathrm{c}$ can be obtained recursively as

$r_{2^{i}}=t_{2^{i-1}}\left(1-r_{2^{i-1}} r_{2^{i-1}}\right) r_{2^{i-1}} t_{2^{i-1}}$

and

$t_{2^{i}}=t_{2^{i-1}}\left(1-r_{2^{i-1}} r_{2^{i-1}}\right)^{-1} t_{2^{i-1}}$.

This layer doubling method is highly computationally efficient because the computational time needed for the calculation of a $M$-atomic-layer system, $\log _{2} M$, scales sublinearly with the number of atomic layers. 

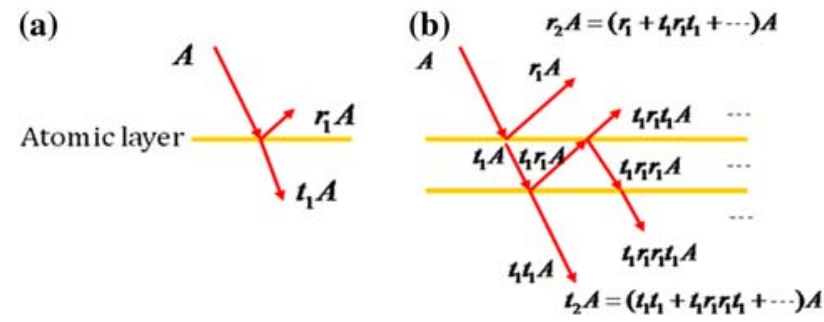

(c)

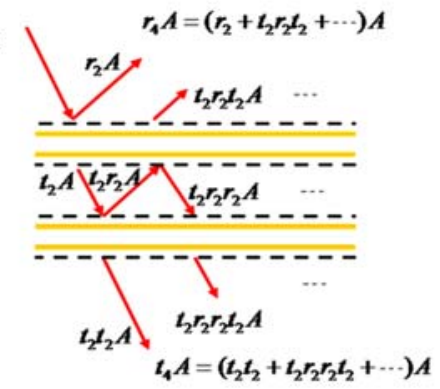

Fig. 4 Schematic illustration of the basic idea of layer-doubling method. a The transmission and reflection coefficients of one atomic layer are $t_{1}$ and $r_{1}$, respectively. $A$ is the wave amplitude of incident electron beam. The wave amplitudes of the transmitted and reflected beam are $t_{1} A$ and $r_{1} A$, respectively. b The transmission $\left(t_{2}\right)$ and reflection $\left(r_{2}\right)$ coefficients of two atomic layers can be obtained by considering the multiple scattering between the two layers. $\mathbf{c}$ The four-layer system can be viewed as a stack of two superlayers and each of superlayer consists of two atomic layer. So the transmission and reflection coefficients of four layer system can be obtained in the same way as the two-layer as long as $t_{2}$ and $r_{2}$ are known

Another bottleneck of the computational efficiency remained in the layer doubling method was the matrix inversion of $\left(1-r_{2^{i}} r_{2^{i}}\right)$, which has to be performed at every recursive step, and whose computation time is scaling cubically with the matrix dimension of the reflection coefficient. Pendry proposed a method to get around this bottleneck based on a perturbation expansion, recognizing that the strong forward scattering of low energy electrons implies that the reflection coefficient, $|r| \ll 1$. Therefore, the expansion should be in order of powers of the small parameter $r$. However, it was found that a simple perturbation approach failed in giving the converged result at the expense of affordable computational time. A more sophisticated perturbation scheme, the so-called renormalized forward-scattering perturbation theory, was finally developed to solve the problem.

With these theoretical advances, the calculations of $I-V$ curves were capable to solve surface structures with up to five atoms in a unit cell. In the period from the early 1970s to the mid 1980s, several hundred structures of clean surface and simple adsorption systems were determined by the LEED crystallography. These studies unveiled that the reconstruction is a common phenomenon at the clean surfaces. For example, the top atomic layer of the $\operatorname{Ir}(100)$ surface undergoes a $(5 \times 1)$ reconstruction [37] (Fig. 5a). This structure similar to the close-packed fcc(111) surface lowers the surface energy of the system. The studies of ethylene chemisorption on transition metals such as Pt and $\mathrm{Rh}$ suggested that ethylene is not necessarily laying flat on the surface, and that, at the room temperature, $\mathrm{C}-\mathrm{H}$ bonds may break and reform to produce ethylidyne on the surface [38, 39] (Fig. 5b).

As the complexity of the surfaces increased so did the computational resources required to perform both the LEED calculations and the fitting of the calculated $I-V$ curves to the experimental data. By the mid of 1980s, it became crucial for the field to develop more computationally efficient methods for data analysis of disordered adsorption systems and reconstructions involving multiple surface layers.

For disordered adsorption systems, a surface unit cell has effectively infinite area (or says, infinite number of atoms). To tackle this problem, diffuse LEED (DLEED) theory was developed by Pendry [40] and Van Hove [41], separately. In the Van Hove's method (known as Beam Set Neglect method), the disordered adsorption surface is approximated by an ordered structure with a unit cell area less than $\lambda^{2}$, here $\lambda$ is the mean free path of electron in the solid (typically around 10-100 $\AA$ ). The physics behind this approach is that the low energy electrons have a relatively short mean free path, and that an electron can only contribute to the diffraction pattern if it has traveled a distance of less than the mean free path. Pendry's approach is based on the observation that a disordered adsorption system can be viewed as a disordered overlayer of atoms adsorbed on an ordered substrate. The electrons scattered from the ordered substrate generate the Bragg spots. Any electron contributing to the diffuse component of the pattern must have interacted with at least one adsorbed atom. Depending on the traveling path taken by the diffracted electrons, the diffraction pattern can be broken into three components, which could be computed using either conventional LEED theory or methods borrowed from the theory of surface extended X-ray absorption fine structure spectroscopy (SEXAFS). With the help of DLEED theory, the structures of weakly adsorbed molecules such as benzene on $\mathrm{Pt}(111)$ could be determined [42] (Fig. 6). The benzene structure unveiled that the preferred adsorption site is the bridge site on $\operatorname{Pt}(111)$, and that the adsorption also induces subtle restructuring of the benzene molecules.

The development of tensor LEED theory by Rous and Pendry [43] finally brought the LEED technique into its mature state. Tensor LEED is a perturbative approach to the calculation of LEED intensities. One starts by defining a reference structure: a particular surface structure that we guess to be as close as possible to the actual surface structure. We then distort this surface by moving some of 
Fig. 5 a The structure of the $(5 \times 1)$ reconstructed $\operatorname{Ir}(100)$ surface. $\mathbf{b}$ The structure of ethylidyne on the $\operatorname{Pt}(111)$ surface (a)

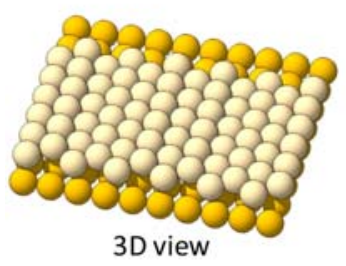

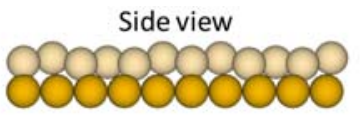

Top view

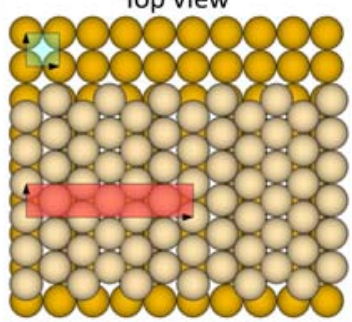

(b)

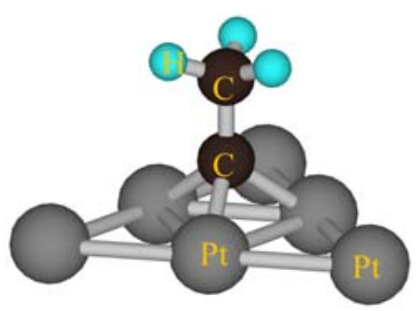

(a)

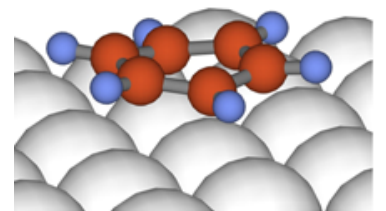

(c)

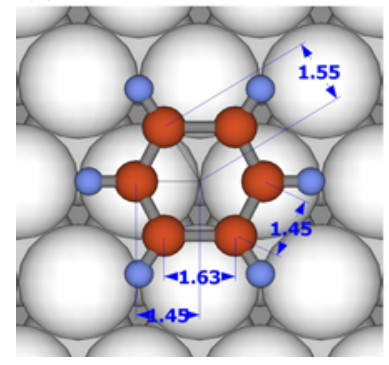

(b)

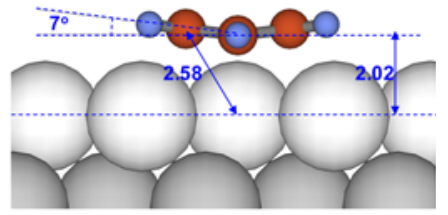

(d)

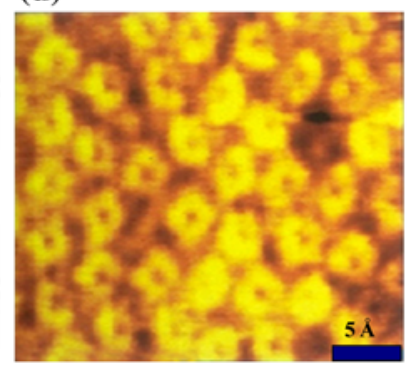

Fig. 6 a-c The structure of weakly bonded benzene molecule on $\mathrm{Pt}(111)$ resolved by diffuse LEED. d STM image of the disordered benzene layer on the $\mathrm{Rh}(111)$ surface [94]

the atoms to new positions. In this way we generate a trial structure that is a structural distortion of the reference structure related by a set of atomic displacements.

If the atomic displacements are small enough (typically within $0.4 \AA$ ), the difference between the amplitude of a given LEED beam scattered from the reference and the trial surface, $\delta A$, can be approximated to the first order. Assuming $\delta r_{n i}(n=1,2, \ldots, N ; i=1,2,3)$ are the $3 \mathrm{D}$ displacements of $N$ atoms, the amplitude difference can be written by

$\delta A=\sum_{n=1}^{N} \sum_{i=1}^{3} T_{n i} \delta r_{n i}$.

The quantity $\mathbf{T}$ is the tensor which depends only on the scattering properties of the reference surface and can be calculated once by the conventional multiple LEED theory. Once $\mathbf{T}$ is known, then the diffraction intensities for many trial surfaces can be evaluated extremely efficiently by summing Eq. 5. This linear version of tensor LEED is limited to atomic displacements of less than $0.1 \AA$. A more

sophisticated version of the theory allowed the displacements of up to $0.4 \AA$. Figure 7 shows the tensor LEED approach which combines the experimental measurement and the theoretical data analysis.

Tensor LEED represented a revolution in structural surface chemistry. The knowledge accumulation of tensor LEED studies leads to the concept of 'flexible surface' which changed our static view of surface structure to a dynamic one. The relaxation at $\operatorname{Pt}(210)$ stepped surface involves the displacements of atoms in up to four surface layers [44] (Fig. 8a), and the marked restructuring of metal surfaces may be induced by strong chemisorption as shown in the cases of the ethylene adsorption on the $\operatorname{Pt}(111)$ [45] and $\mathrm{Rh}(111)$ [46] surfaces (Fig. 8b). The creative applications of tensor LEED to the covalent-bonded and ionicbonded materials such as $\mathrm{NaCl}$ [47] and ice [48, 49] further proved the generality of the concept of 'flexible surface' (Fig. 9a, b).

Recently, the structure studies of nanostructures pose another challenge to the LEED technique. It can be envisioned that, with the advances both in new experimental design and theoretical data analysis, this technique will become one of the prime tools for determining complex structures of nanostructures in near future [50].

\section{Structure and Stability of Surface Oxides}

In recent years, the formation of thin well-ordered but complex surface oxides on later transition metals has been discovered [51]. These surface oxides may serve as a protective layer against corrosion, as insulation layers in microelectronic devices, and as oxygen reservoir during catalytic reactions. Due to the structural complexity of these surface oxides, a multi-method approach of experimental and theoretical techniques has to be employed in the atomic scale studies. These studies provide perfect examples for demonstrating the complementary roles of experimental and theoretical techniques in surface chemistry studies. 
Fig. 7 Scheme showing the tensor LEED approach in determining the complex surface structure

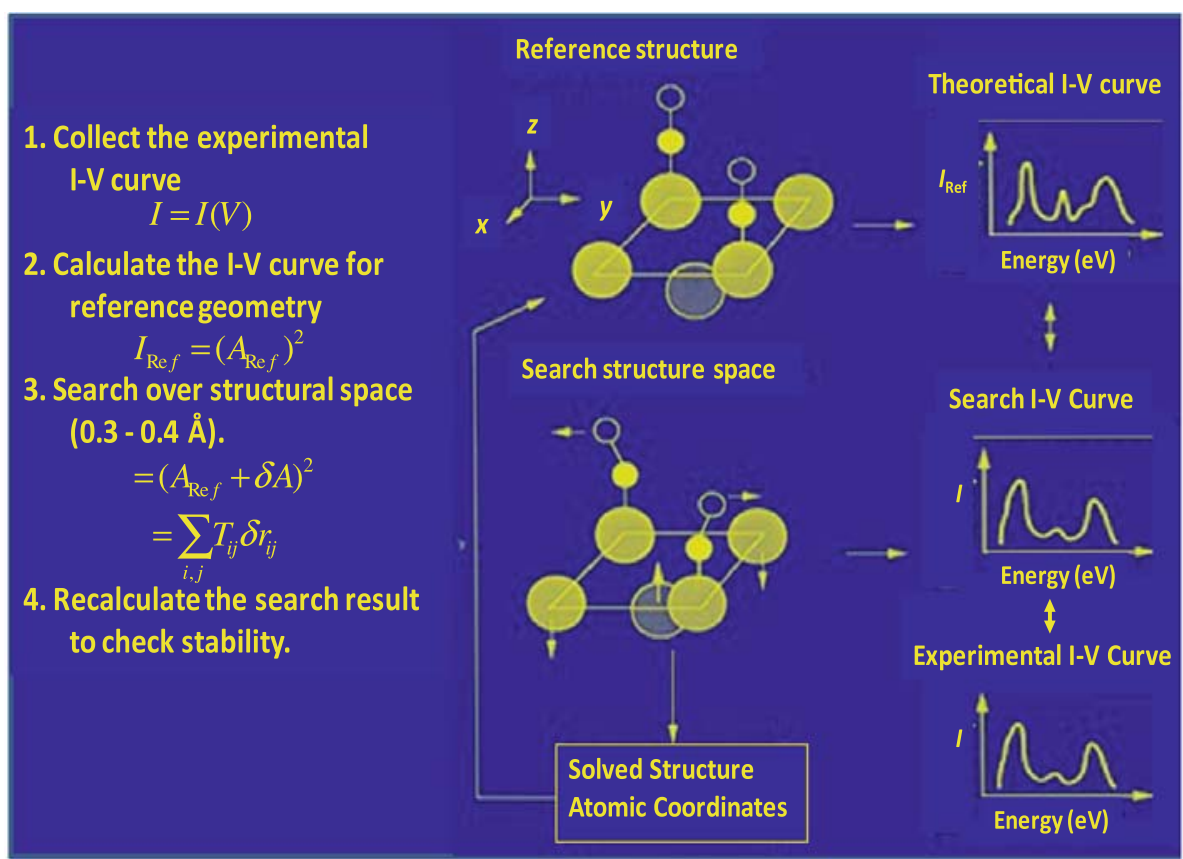

(a)

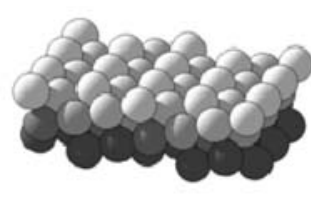

30พับ

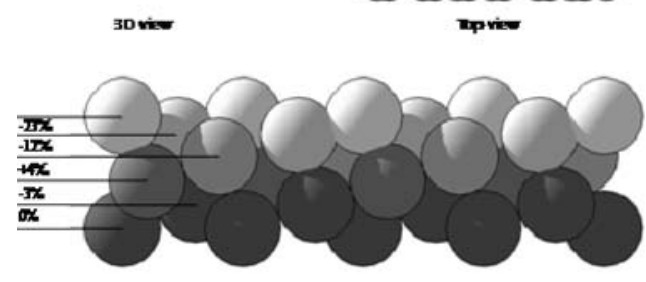

Site vis

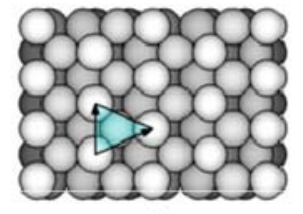

Side view

(b)
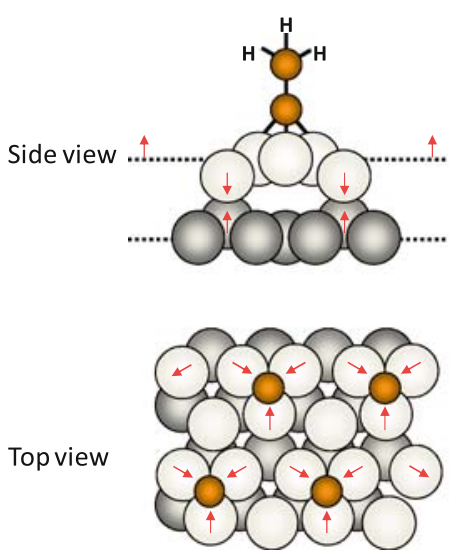

$\operatorname{Rh}(111)-(2 \times 2)-\mathrm{C}_{2} \mathrm{H}_{3}$ hcp site
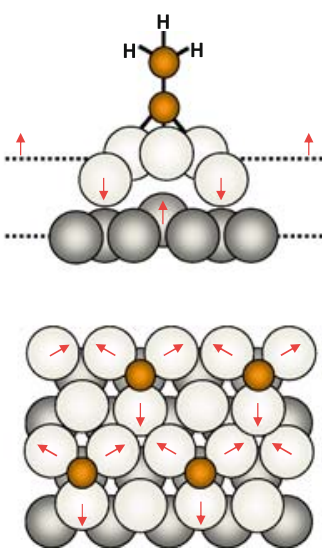

Fig. 8 a Multilayer relaxation of $\operatorname{Pt}(210)$ surface determined by the tensor LEED. b Restructuring of $\operatorname{Pt}(111)$ and $\operatorname{Rh}(111)$ surfaces induced by ethylene chemisorptions

The multi-method approach starts with applying the qualitative structural methods such as LEED and STM. These two techniques give a good approximation of the symmetry of surface structure and in-plane lattice distances. Figure 10a and $\mathrm{b}$ shows the LEED pattern and the STM image of a surface oxide on $\mathrm{Rh}(111)$ formed under conditions: $1 \times 10^{-3}$ mbar of $\mathrm{O}_{2}$ and $700 \mathrm{~K}$ [52]. These experimental results suggest the formation moiré pattern consisting of a hexagonal layer with a larger in-plane lattice distance being on top of hexagonal $\mathrm{Rh}(111)$ substrate. The periodicity of the oxygen-induced hexagonal pattern is close to a $(9 \times 9) \operatorname{Rh}(111)$ cell. The lattice distance of the overlayer is around $3 \AA$, which can also be confirmed by using surface X-ray diffraction (SXRD) measurement.

Applying high resolution core level spectroscopy (HRCLS), a type of XPS technique, the chemical composition of surface oxides can be studied quantitatively. For the Rh $(9 \times 9)$ surface oxide (Fig. 10c), the HRCLS spectrum in O1s region indicates there are two $\mathrm{Rh}$-coordinated $\mathrm{O}$ species existing in the surface oxide layer; In the $\mathrm{Rh} 3 \mathrm{~d}_{5 / 2}$ region, there are two major peaks. The peak at higher binding energy $(\sim 307.9 \mathrm{eV})$ is originated from a highly-O-coordinated Rh species. The abundances of these surface species can be deducted qualitatively from their peak intensities in the HRCLS spectra. The obtained 


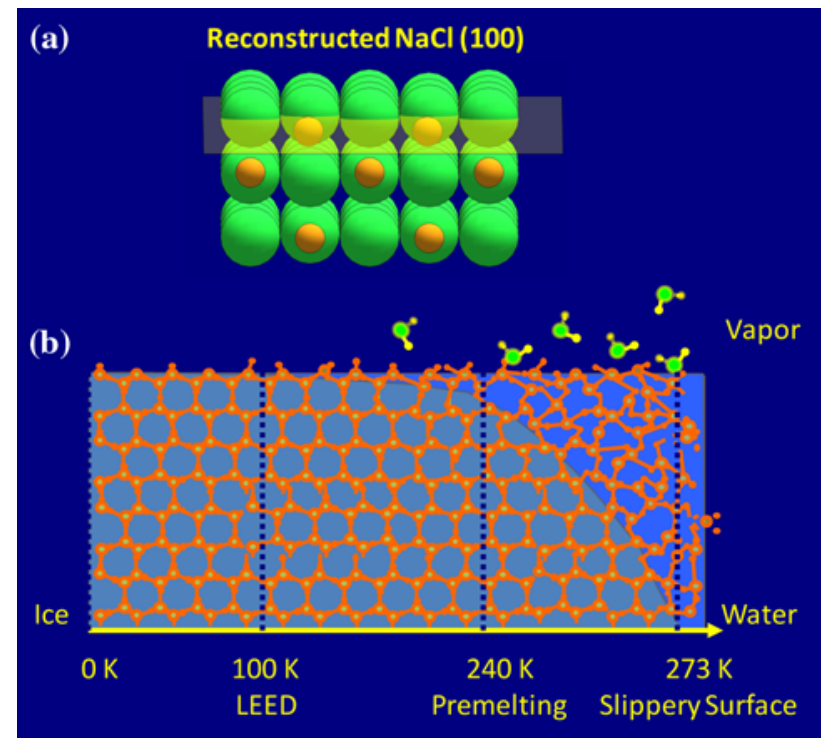

Fig. 9 Schematic illustrations of a the reconstructed $\mathrm{NaCl}(100)$ surface and $\mathbf{b}$ the ice(0001) surface at different temperatures. The LEED results indicate that the surface $\mathrm{Na}^{+}$layer move towards the bulk. The LEED results on the ice(0001) surface suggest that the surface is terminated by a full bilayer of water molecules, and, even at $100 \mathrm{~K}$, the surface root-mean-square vibrational amplitude is two to three times larger than that in the bulk

coverages for the highly $\mathrm{O}$-coordinated $\mathrm{Rh}$ species and the $\mathrm{Rh}$-coordinated $\mathrm{O}$ species are 0.9 and 1.8 monolayer, respectively, which indicates a layered $\mathrm{O}-\mathrm{Rh}-\mathrm{O}$ surface oxide.

Using the experimentally obtained structural information such as symmetry and the abundances of surface species, the atomic structural models can be proposed and examined by the DFT studies. In the DFT studies, a O-RhO trilayer with a $(7 \times 7)$ cell on a $(8 \times 8) \mathrm{Rh}(111)$ cell (Fig. 11a, b) is found to be stable at the given oxygen partial pressure and temperature [52]. The simulated STM image (Fig. 11c) for this structure is in good agreement with the experimental result shown in Fig. 10b; further, the calculated core electron binding energies agree well with the measured values as shown in Fig. 10c. The DFT-predicted structure disagrees slightly with the SXRD result which suggests a structure with a $(8 \times 8)$ cell on a $(9 \times 9)$ $\mathrm{Rh}(111)$ cell. However, DFT calculations also indicate that the free energy difference between these two structures is very small.

Once the structures are obtained, the thermal and chemical stability of surface oxides can be investigated in detail. The calculated phase diagram of various surface oxides indicates that the $(8 \times 8)$ and the $(9 \times 9) \mathrm{Rh}$ surface oxides are actually metastable under the conditions where bulk oxide is already stable [52]. Therefore, these oxides serve as kinetic barriers for the further growth of thick oxides on the surface.

The investigation of the reduction of the $(9 \times 9) \mathrm{Rh}$ surface oxide by $\mathrm{CO}$ at $\mathrm{CO}$ partial pressure of $2 \times 10^{-8}$ mbar and $375 \mathrm{~K}$ found that the surface oxide can be reduced even though $\mathrm{CO}$ does not adsorb easily on the surface under the given experimental conditions [53]. Both HRCLS and STM results showed that atomic oxygen is expelled from the oxide layer onto the reduced metallic areas. The observations can be again explained by the DFT calculations. The DFT result showed that the $(9 \times 9)$ structure is not stable, if its surrounding metal is free of oxygen. Therefore, the surface oxide may serve as an oxygen reservoir during the $\mathrm{CO}$ oxidation reaction.

\section{Surface Chemical Bond}

Once the geometric structures of chemisorption systems are determined by various surface science techniques, the further questions are how strong these surface chemical bonds are, and how the strength of the surface chemical bond depends on the properties of the adsorbed molecules and the substrates. In experiments, the strength of surface chemical bonding can be determined by deriving the heat of adsorption from the adsorption isotherms at different temperatures, or by monitoring desorption temperature of adsorbate in the temperature-programmed desorption (TPD) experiment. By the late of 1970s, large amount of experimental data had been accumulated, and the surface chemical bonding strength across the periodic table was
Fig. 10 a The LEED, and $\mathbf{b}$ the STM image of a surface oxide formed on $\mathrm{Rh}(111)$ at $1 \times 10^{-3}$ mbar of $\mathrm{O}_{2}$ and 700 K. c The XPS spectra of the oxide surface. The calculated core electron binding energies of different $\mathrm{O}$ and $\mathrm{Rh}$ species are also shown as the vertical lines for comparison (a)

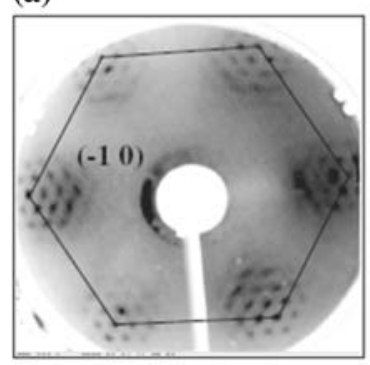

(b)

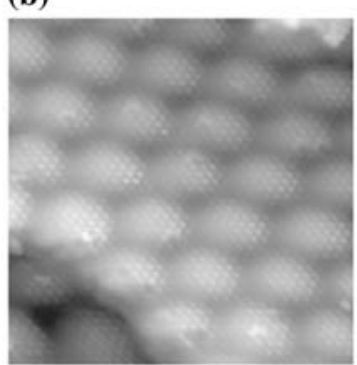

(c)

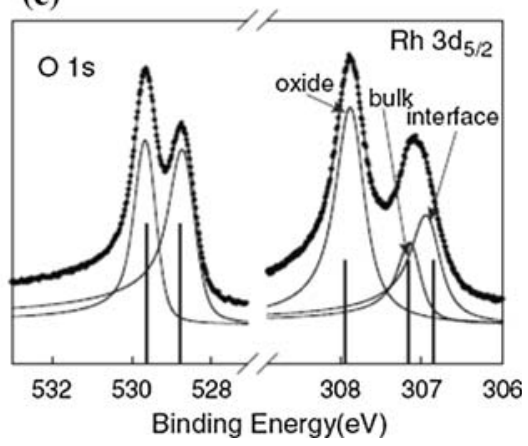


Fig. 11 a, b The DFT predicted O-Rh-O trilayer structure of the surface oxide on $\mathrm{Rh}(111)$. $\mathrm{c}$ The simulated STM image of the surface oxide (a)

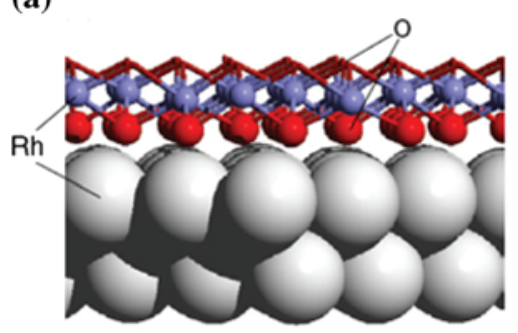

(b)

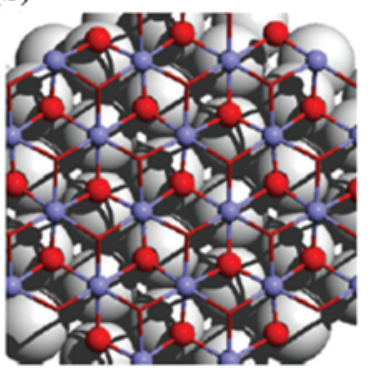

(c)

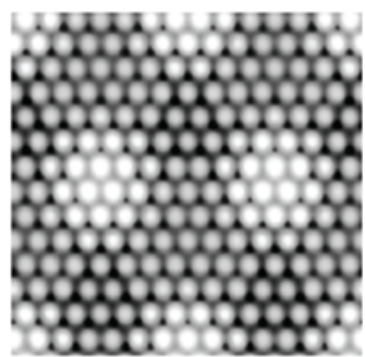

tabulated [54]. It was found that, over transition metal surfaces, the chemical bonding strength of an adsorbed atom generally increases from the right to the left in the periodic table. On the other hand, the development of the electron spectroscopy techniques, such as ultraviolet photoelectron spectroscopy (UPS) [55-57] and X-ray photoelectron spectroscopy (XPS) [58, 59], enabled the detailed investigation of electronic structures of chemisorptions systems [60]. All these experimental advances set a stage for the development of theoretical approaches to rationalize the experimental observations, and to understand how the electrons in the adsorbates and the metal surface interact with each other to form surface chemical bonds.

A major contribution by Norskov in the early stage of this theoretical development was extending and applying the effective medium theory to understand the trends of chemical bonding over the transition metal surfaces $[13,61,62]$. The effective medium theory is based on density functional theory (DFT), a general theory for studying molecular electronic structures. The full-blown DFT study of surface chemical bonding is very time consuming due of the large number of electrons involved. The basic idea behind the effective medium theory is to calculate the energy of an atom in an arbitrary environment by first calculating it in some properly chosen reference system, the effective medium, and then estimate the energy difference between the real system and the reference system [62]. The total energy of the system is given by

$E=\sum_{i} E_{c, i}+\left(E-\sum_{i} E_{c, i}\right)$

where $E_{c, i}$ is the energy of atom $i$ in the reference system. The essence of the method is then to choose the reference system so close to the real system that the correction, $\Delta E=E-\sum_{i} E_{c, i}$, is small enough that it can be estimated using perturbation theory or some other approximation form. The choice of the reference system also ensures that the binding energies of the reference system, $E_{c, i}$, can be easily obtained.

In the simplest form, the adsorbed atom is considered to be embedded in a homogenous electron gas (the reference system) with an average electron density corresponding to

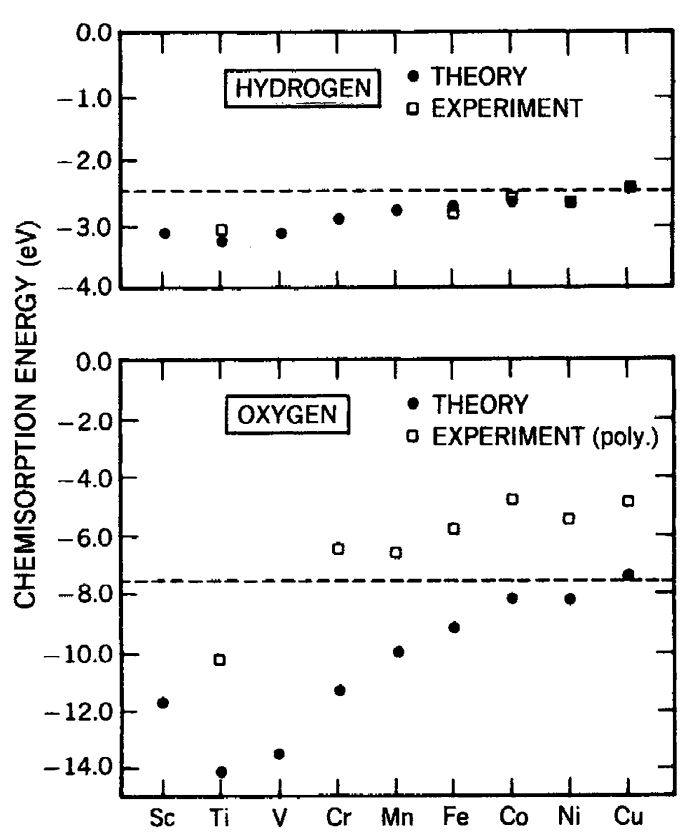

Fig. 12 The chemisorptions energies of hydrogen and oxygen across the periodic table. The hollow squares are the experimental results. The black dots are the results predicted by the effective medium theory

the given metal. The binding energy of each atom is calculated to the first order of approximation as a function of the average electron density from its neighbors in the vicinity of the atom. The correction $\Delta E$ is calculated by the News-Anderson model which considers subsequent interaction of the valence electron of adsorbed atom with the $s p$ bands and the $d$ band in the metal. It turned out that, as shown in Fig. 12, this simple treatment was good enough to predict the bonding trends observed experimentally for the chemisorption of hydrogen and oxygen over the transition metal surfaces [13].

The further refinement of the effective medium theory by Norskov and coworkers leads to a simple yet powerful theory, the $d$-band model [14-16], for understanding the variations of chemisorptions energy from one to another metal, from one surface structure to another on the same metal. In the $d$-band model, the adsorption energy is given by [63] 
$\Delta E=\Delta E_{0}+\Delta E_{d}$

where $\Delta E_{0}$ is the bond energy contribution from the freeelectron-like $s p$ electrons and $\Delta E_{d}$ is the contribution from the extra interaction with the transition metal $d$ electrons. It is assumed that $\Delta E_{0}$ is independent of the metal. $\Delta E_{d}$ can be calculated by the News-Anderson model as

$\Delta E_{d}=\alpha\left|V_{a d}\right|^{2}-2(1-f) \frac{\left|V_{a d}\right|^{2}}{\varepsilon_{d}-\varepsilon_{a}}$

where the first term is the Pauli repulsion between the adsorbate states and the metal $d$ states, which is proportional to $\left|V_{a d}\right|^{2}$, the square of the coupling matrix element between the adsorbate states and the metal $d$ states. The second term is the attraction contribution from the hybridization of the adsorbate states and the metal d states. The hybridization leads to a bonding orbital below the Fermi level and an antibonding orbital close to the Fermi level as shown in Fig. 13. Just like the situation in the chemical bonding between two atoms, the strength of the surface chemical bond is determined by the occupancy of the antibonding orbital. The number of electrons in the antibonding orbital is approximately equal to the initial filling of the $d$ band of the free metal surface. $\varepsilon_{d}$ and $\varepsilon_{a}$ are the energy at the center of the metal $d$ band and the adsorbate states, respectively.

When comparing the chemisorptions energies of a given molecule on different metals, the $d$-band model suggests that the adsorption energy variations are mainly due to the changes of $V_{a d}$ and $\varepsilon_{d}$. Figure 14 shows variations of the $\mathrm{O}$ adsorption energy over the $4 \mathrm{~d}$ transition metals [16]. The results of the simple $d$ band model are in good agreement with that from the full DFT calculations and the experiments. It also shows that the adsorption energy increases as

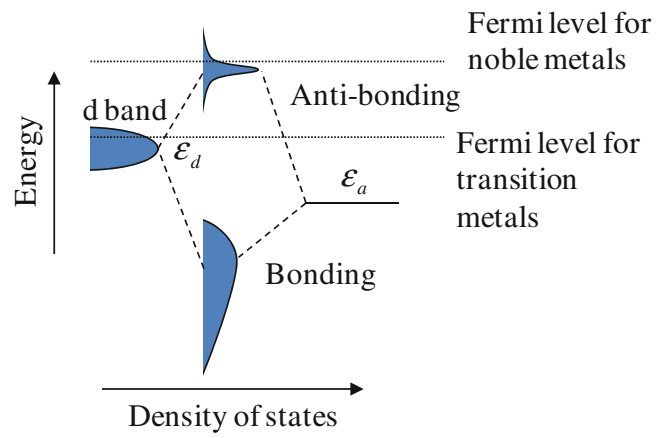

Fig. 13 The hybridization of the metal $d$ band, $\varepsilon_{d}$, with the adsorbate state, $\varepsilon_{a}$, to form the bonding and the antibonding orbitals. The electron occupancy of the antibonding orbital determines the bonding strength. The higher the occupancy, the weaker the surface chemical bond is. For noble metals, the antibonding orbital is fully filled because the $d$ band is deep below the Fermi level and fully filled initially. For transition metals, the $d$ band is not fully filled initially. So, after the formation of chemisorption bond, the antibonding orbital is partially filled

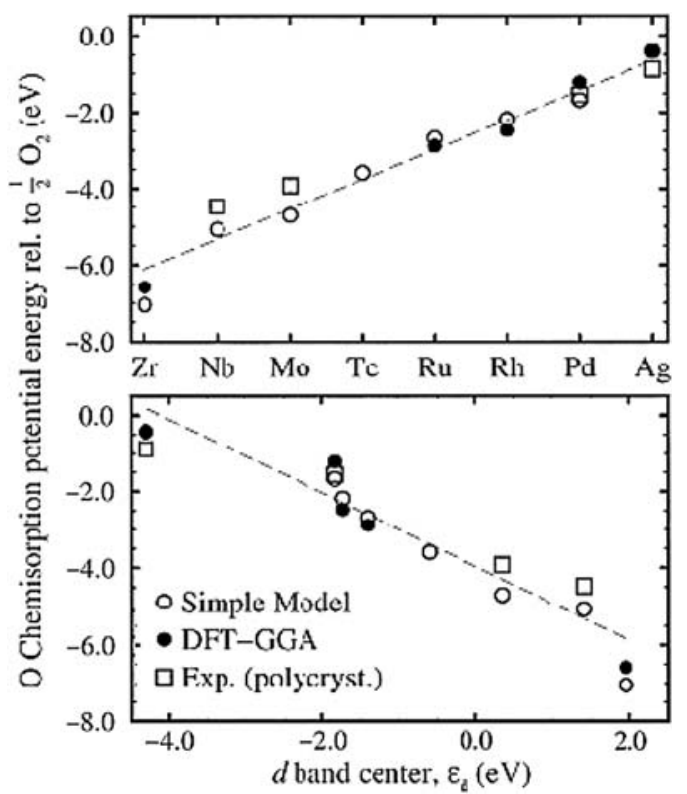

Fig. 14 The oxygen adsorption energies along the $4 \mathrm{~d}$ transition metal series (the upper panel). The adsorption energies are plotted as a function of the $d$-band center energy on clean metal surfaces (the lower panel)

the $d$ band center shifts up to the Fermi level and the $d$ band becomes less filled.

It was observed experimentally that the adsorbed atoms and molecules have higher heats of adsorption at defect sites such as the steps and kinks on the surface [64] (Fig. 15a). The calculations by DFT show that the $d$ band centers at the defect sites shift up relative to the sites on the flat surface, which leads to the increase of the adsorption energy [65] (Fig. 15b). Using the same argument, the $d$ band model has been applied to explain and predict the alloying effect on the chemisorptions observed in the experiment. The examples shown in Figs. 14 and 15 clearly demonstrate that the simple $d$-band model captures the main factors that determine the chemisorption energies of atoms and small molecules on the transition metal surfaces.

For more complex chemisorption systems in which adsorbates can form multiple bonds with several surface atoms, a scaling relation has been proposed recently based on extensive DFT calculations of adsorption energies of $\mathrm{CH}_{x}$ species on the metal surfaces [66]. Figure 16 shows that, for a given $x$, the adsorption energies of $\mathrm{CH}_{x}$ on different metal surfaces is scaled almost linearly with the atomic adsorption energies of carbon, which implies a scaling relation

$\Delta E^{A H_{x}}=\gamma(x) \Delta E^{A}+\xi$,

here $\gamma(x)$ and $\xi$ are fitting constants. From the fitting constants shown in Fig. 16, we can further see that the values of $\gamma(x)$ is very close to that predicted by 


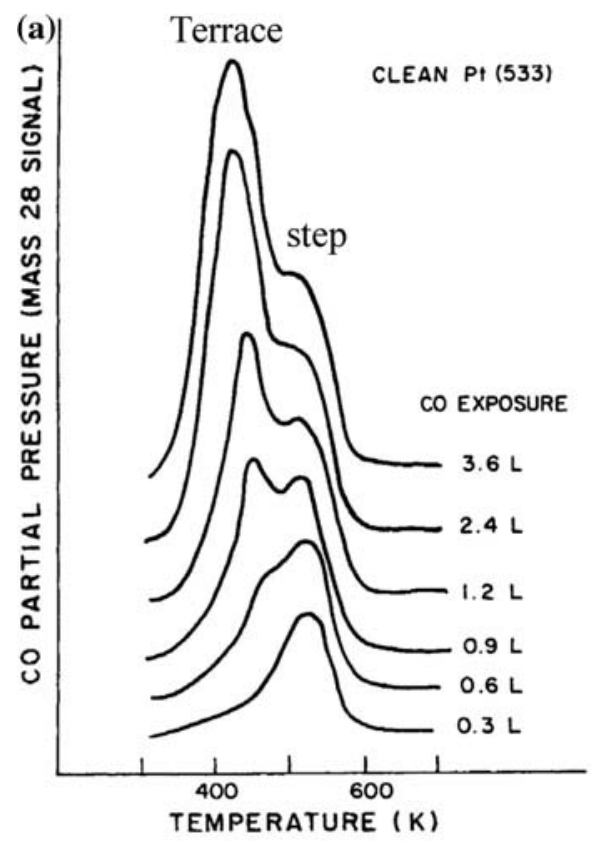

Fig. 15 a TPD results of $\mathrm{CO}$ on the stepped $\mathrm{Pt}(533)$ surface at different coverages. There are major desorption peaks at relative higher coverages. By comparing to the TPD results of $\mathrm{CO}$ on the flat $\mathrm{Pt}(111)$ surface, the peak at the lower temperature can be attributed to the desorption of CO adsorbed on the (111) terrace. The peak at the higher temperature is due to the $\mathrm{CO}$ on the step sites. Because of the

$\gamma(x)=\frac{x_{\max }-x}{x_{\max }}$,

here $x_{\max }$ for carbon atom is 4 , that is the maximum number of bonds carbon can form with the surface atoms. This scaling relation, which has been also observed in several other chemisorptions systems [19], provides a semi-quantitative method to predict the adsorption energies of complex adsorbates from the simple calculation of atomic adsorption energy.

\section{Reactivity and Selectivity in Heterogeneous Catalysis}

Heterogeneous catalytic reactions involve elementary processes: adsorption and dissociation of reactants from the gas phase, diffusion of surface species, surface reactions to form surface intermediates and products, and desorption of products into the gas phase. The ultimate goal of surface science research is to obtain the molecular level details of these elementary processes, and to control reactivity and selectivity of catalytic reactions by using the obtained molecular level knowledge. Apparently, neither experimental study nor theoretical study can fulfill this endeavor alone. The capability of experimental study is always limited by the spatial, time, and energy resolutions achievable by experimental techniques. For example, (b)

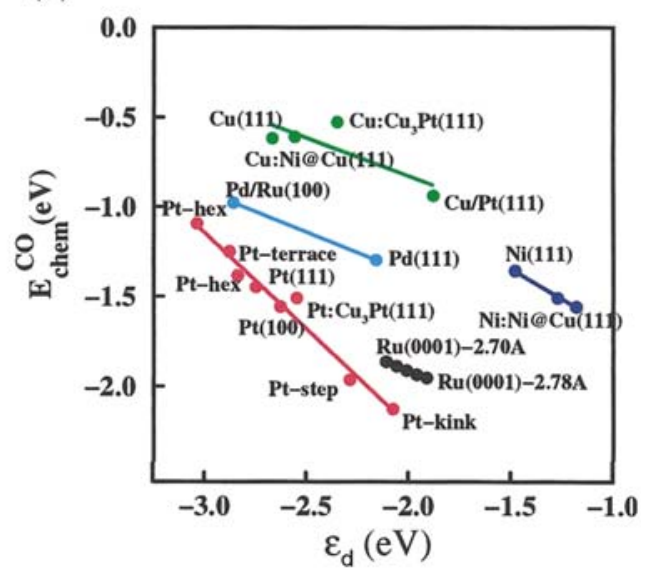

higher adsorption energy at the step sites, $\mathrm{CO}$ molecules preferentially occupy these step sites at the low coverages. b CO chemisorption energies on the flat, stepped, strained, and kinked metal surfaces calculated by the $d$-band model. Theory model indicates the up-shift of the $d$-band center at the defect sites causes the increase of chemisorption energy

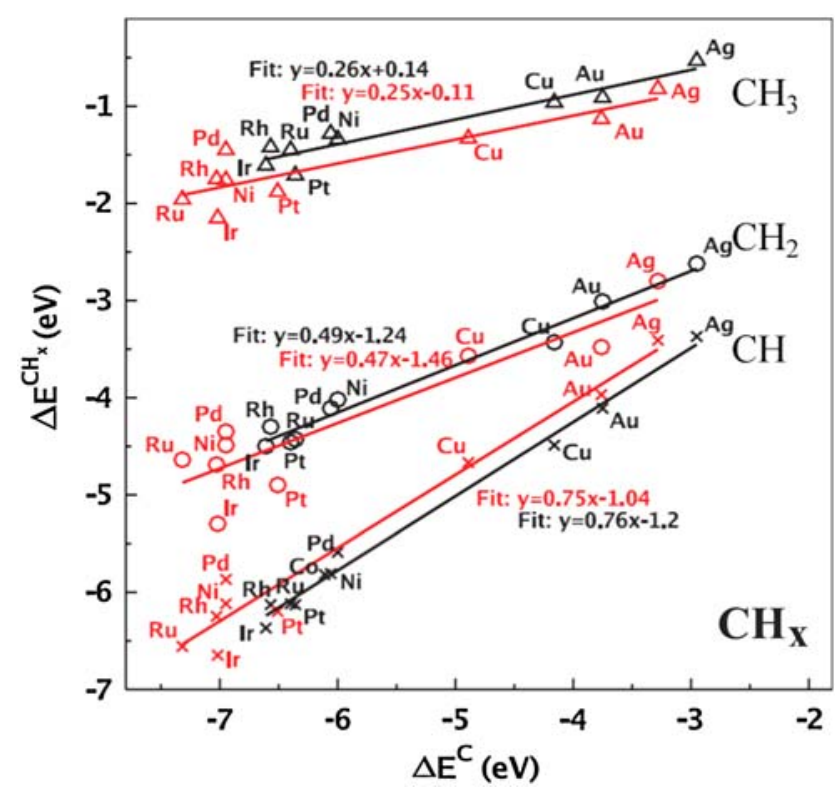

Fig. 16 Calculated adsorption energies of $\mathrm{CH}_{x}$ intermediates as a function of the adsorption energies of atomic $\mathrm{C}$ on the flat and stepped surfaces of various metals. The red lines are for the stepped surface, and black lines for the flat surfaces. For a given $x$, the adsorption energies of $\mathrm{CH}_{x}$ can be fitted by a straight line, $\Delta E^{C H_{x}}=\gamma(x) \Delta E^{C}+\xi$

monitoring the surface intermediates during catalytic hydrocarbon conversion under the realistic reaction conditions has been proved to be extremely difficult; on the 
theoretical side, theoretical model usually tends to oversimplify the local chemical environment in which the elementary reaction processes take place. The complexity of the local chemical environment includes the coadsorption of surface species and their coverages on the catalyst surface, the distribution of active surface sites, etc. Therefore, combining experimental and theoretical approaches is a must in the molecular level study of catalytic reactions.

A recent study of ammonia synthesis over a ruthenium nanoparticle catalyst by Norskov and coworkers demonstrated how the theoretical modeling and experimental techniques can complement each other to achieve the molecular level understanding of this simplest catalytic reaction under industrially relevant reaction conditions [67, 68]. In this study, the potential energy diagram for the full reaction was constructed based DFT calculations. The activation barriers for the reactions taking place on the terrace site and the step site were compared (Fig. 17). It was shown that the dissociation of nitrogen (the rate limiting step) on the step site has a much lower activation barrier than that on the terrace site. The step site is the active site for this reaction. The potential energy diagram also provided all necessary information to calculate the rates of the individual elementary steps in the catalytic reaction by the micro-kinetic model. In the calculations of the dissociative adsorption rate of $\mathrm{N}_{2}$, the coadsorption effect was also considered by investigating the activation energy changes induced by coadsorption of atomic nitrogen or hydrogen. In parallel to the theoretical study, the ruthenium nanoparticle catalyst was synthesized and the particle size distribution and the surface morphology of nanoparticles were investigated by transmission electron microscopy (TEM) experiment (Fig. 18a, b). Based on the TEM results, the number of active sites per gram of catalyst can be estimated. Using the experimentally obtained active site concentration, the $\mathrm{NH}_{3}$ productivity from a plug flow reactor loaded a catalyst containing $0.2 \mathrm{~g}$ of the $11.1 \%$ $\mathrm{Ru} / \mathrm{MagAl}_{2} \mathrm{O}_{4}$ catalyst was calculated under realistic reaction conditions. The agreement between experimental and theoretical results shown in Fig. 18c is surprisingly well considering the complexity of the catalytic reaction over the nanoparticle catalyst.

The success of this study also gives hope to develop theoretical computer-based method into a indispensable tool in rational design of catalyst. One of major bottlenecks for the computational study of reactivity and selectivity in heterogeneous catalysis is identifying the transition states of surface reactions and computing the activation energies. Fortunately, there are some empirical relations correlating the activation energy and reactivity with the chemisorption energy of reactants. One of them, the Brønsted-EvansPolanyi (BEP) relation [69-72], states that the activation energy for an elementary reaction step on surface depends linearly on the reaction energy, that is, the difference between the chemisorption energy of the products and the reactants. An example [72] for the activation energies for $\mathrm{N}_{2}$ dissociation over various metal surfaces are shown in Fig. 19a. Another relation is the famous principle of Sabatier [73]: the best catalyst is one that binds the intermediates not too strongly and not too weakly. Figure $19 \mathrm{~b}$ shows how the rates of ammonia synthesis depends on the nitrogen chemisorption energies on various metal surfaces [74]. These relations offer an efficient way to estimate the
Fig. 17 The calculated potential energy diagram for ammonia synthesis from $\mathrm{N}_{2}$ and $\mathrm{H}_{2}$ over close-packed (001) and stepped Ru surfaces. A * denotes an empty site and $\mathrm{X}^{*}$ an adsorbed species. The solid line is for the reaction on a step site, and the dashed line on the terrace. The configurations of the transition states for $\mathrm{N}_{2}$ dissociation over the terrace and stepped sites are shown in the insets

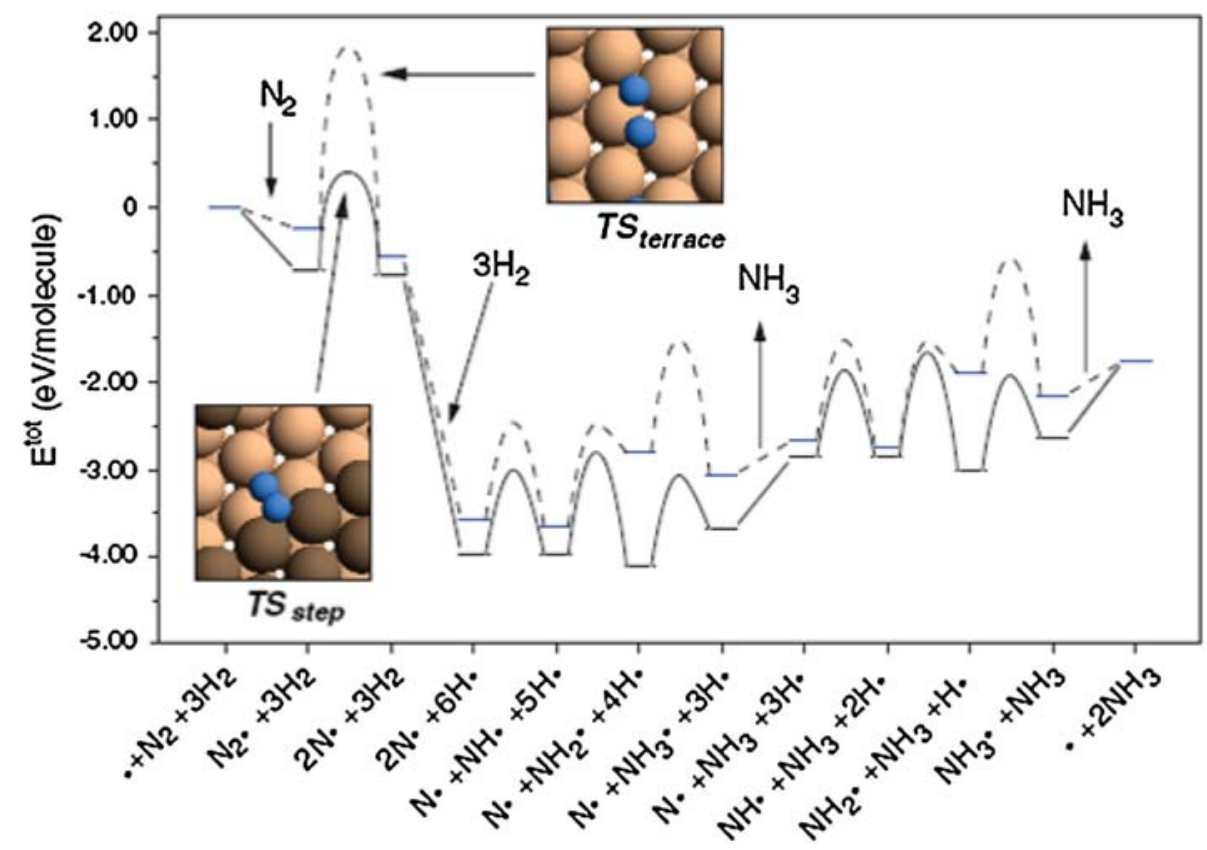


Fig. 18 a High resolution TEM image of a supported ruthenium nanoparticle with a step. b The particle size distribution obtained from the TEM experiments. c Comparison of ammonia productivity from the model with experiment results. The productivity is plotted as a function of the reaction temperature (a)

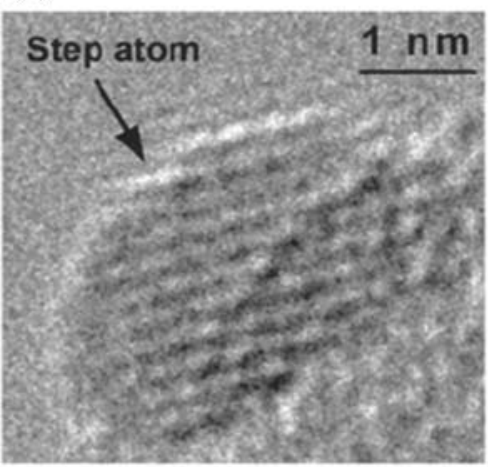

(b)

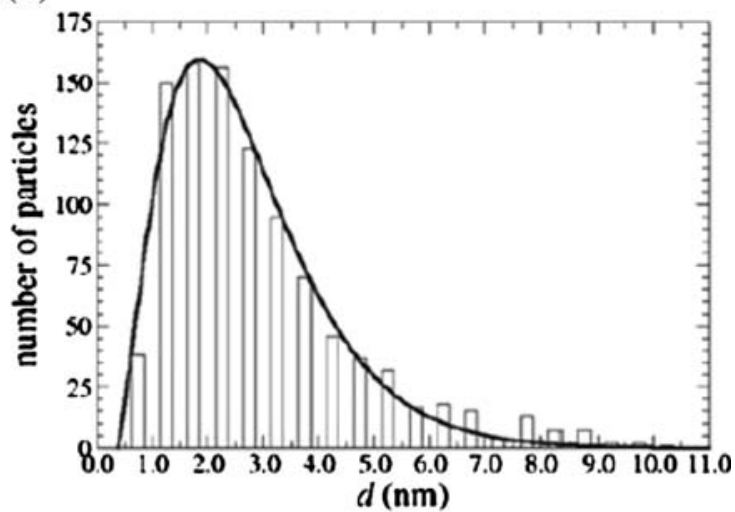

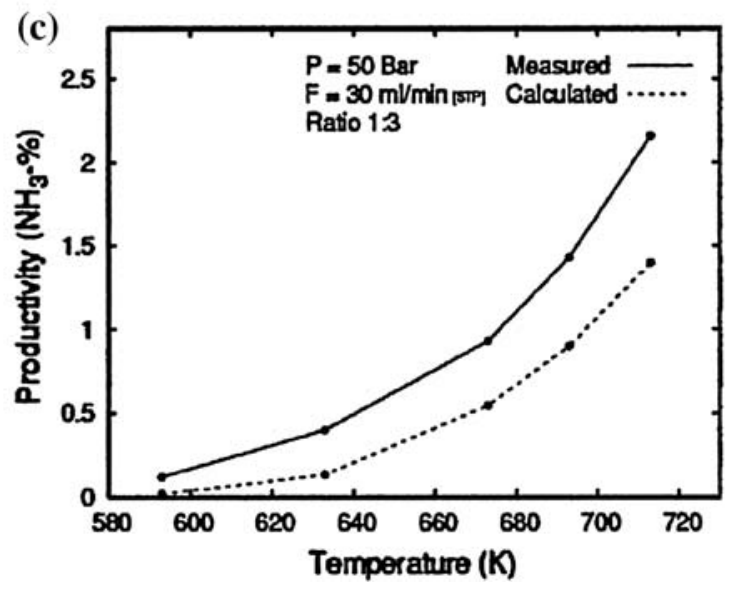

(a)

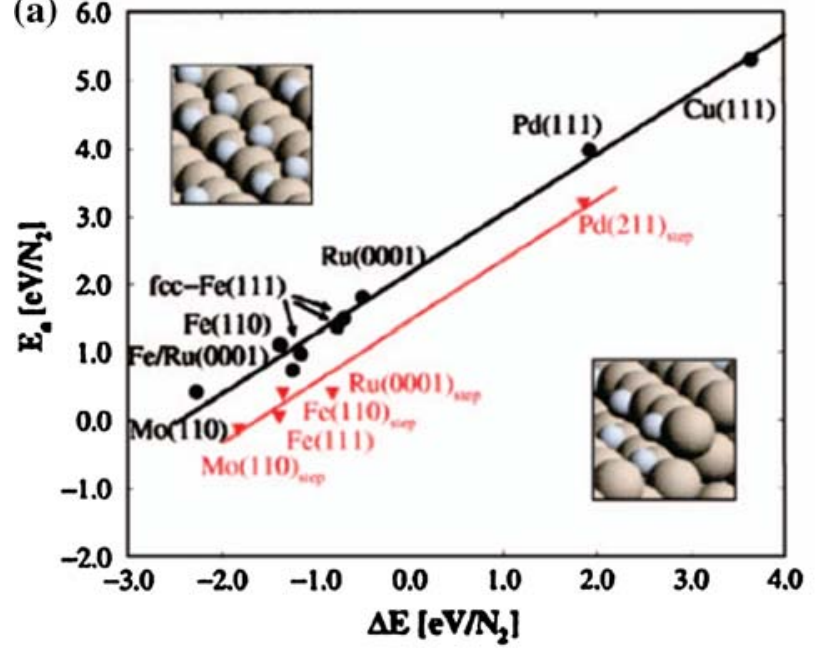

Fig. 19 a The calculated activation energies of $\mathrm{N}_{2}$ dissociation adsorption as a function of the chemisorption energies of nitrogen on the flat and stepped metal surfaces. The black dot is for the flat surfaces, and the red triangles for the stepped surfaces. The straight fitting lines indicate the BEP relation is applicable in this reaction. $\mathbf{b}$

activation energy and the reactivity using the chemisorption energies of the reactants and products, since the chemisorptions energies can be computed efficiently by the

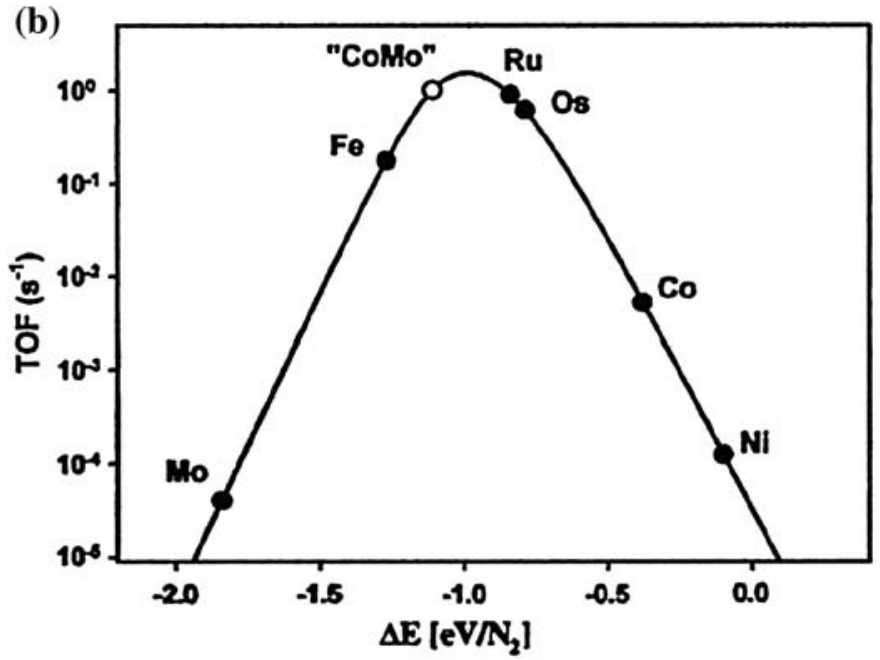

The calculated turnover rates as a function of the chemisorption energies of nitrogen on several metal and alloy surfaces. This chemisorption energy dependence of the reaction rate clearly demonstrates the Sabatier's principle

$d$-band model as we discussed in the previous section. With the help of these relations and the chemisorption model, catalytic properties of alloy combinations can be 
investigated computationally in the search for the low-cost yet highly activity and selective catalysts [27, 74-79].

\section{Summary and Outlook}

We have shown, by examples, the importance of experiment-and-theory-combined approaches in the development of experimental techniques in surface science, resolving the surface structures, and studying chemisorption and catalytic reactions. The contributions by Prof. Norskov and coworkers to the chemisorption theory and the computer-based catalyst design have been highlighted with emphasizing their deep appreciation of experimental developments and their extensive collaboration with experimentalists in the effort to achieve the molecular level understanding of complex catalytic processes.

The major challenges of surface science in the twentyfirst century are to explore the unique physical and chemical properties of nanomaterials, and to design new generation of catalytic processes with high reactivity and selectivity. To face these challenges, experimentalists and theorists have to come together, and be aware of the advantages and the disadvantages of each others' techniques. Here we finish our paper with three interesting problems raised in the study of the nanostructured surfaces and catalytic reactivity and selectivity. These problems need attentions from both experimentalists and theorists.

The first problem is regarding SFG vibrational spectroscopy, a prime in situ technique to monitor the orientation and ordering of adsorbates. Recently, a number of studies have applied this technique to the nanostructured surfaces [80-82]. A general observation in these studies is the reduction of the sum frequency signal due to the nanometer scale corrugation on sample surfaces. Moreover, the surface corrugation also makes it difficult to derive the adsorbate orientation from the SFG measurements with different polarization combinations, since the common SFG theory was initially developed for the flat surfaces [83]. Apparently, further experimental and theoretical development of the SFG technique is needed to improve its sensitivity in the nanomaterial studies.

The second problem is concerning the synthesis of alloy catalysts. At present, the computer-based method is capable to perform large scale screening of alloy catalysts for important catalytic reactions [78, 79]. However, the proposed alloy catalysts are not necessarily stable under harsh reaction conditions, especially, when these catalysts are in the form of nanoparticles. On the one hand, in order to optimize the reactivity and selectivity of alloy nanoparticle, certain surface composition is usually required [22, 27, 75]. On the other hand, the surface composition of alloy nanoparticles may change dramatically with the reaction conditions as shown by an ambient pressure XPS study on bimetallic nanoparticles carried out recently at Berkeley [84]. Therefore, the development of new synthesis schemes for producing alloy catalysts with relatively stable surface composition is extremely important to the rational design of catalyst.

Finally, as a third example of the challenges in catalysis science, obtaining information about the nature and conversion of surface reaction intermediates is the key to understanding the selectivity of complex catalytic reactions [85-87]. Performing in situ spectroscopy techniques such as polarization-modulated reflection-absorption infrared spectroscopy (PM RAIRS) and SFG under reaction conditions usually results in complex spectra [81, 88-91]. The development of reliable theoretical methods for predicting the vibrational frequencies of surface intermediates will provide tremendous help in the spectrum interpretation and in determining the coverages of the reaction intermediates that may adsorbed simultaneously on the catalyst surface $[92,93]$.

Acknowledgments This work was supported by the Director, Office of Science, Office of Basic Energy Sciences of the U.S. Department of Energy under Contract No. DE-AC02-05CH11231.

Open Access This article is distributed under the terms of the Creative Commons Attribution Noncommercial License which permits any noncommercial use, distribution, and reproduction in any medium, provided the original author(s) and source are credited.

\section{References}

1. Somorjai GA (1994) Introduction to surface chemistry and catalysis. Wiley, New York

2. Pendry JB (1974) Low energy electron diffraction: the theory and its application to determination of surface structure. Academic Press, London

3. Van Hove MA, Weinberg WH, Chan CM (1986) Low-energy electron diffraction: experiment, theory, and surface structure determination. Springer-Verlag, Berlin

4. Ibach H, Balden M, Lehwald S (1996) J Chem Soc Faraday Trans 92:4771

5. Szeftel J (1985) Surf Sci 152:797

6. Binnig G, Rohrer H (1987) Rev Mod Phys 59:615

7. Binnig G, Rohrer H (1999) Rev Mod Phys 71:S324

8. Sautet P (1997) Chem Rev 97:1097

9. Tersoff J, Hamann DR (1985) Phys Rev B 31:805

10. Shen YR (1989) Nature 337:519

11. Shen YR (2003) The principles of nonlinear optics. Wiley-Interscience, Hoboken

12. Norskov JK, Lang ND (1980) Phys Rev B 21:2131

13. Norskov JK (1990) Rep Prog Phys 53:1253

14. Hammer B, Norskov JK (1995) Surf Sci 343:211

15. Hammer B, Norskov JK (1995) Nature 376:238

16. Hammer B, Norskov JK (2000) Adv Catal 45:71

17. Greeley J, Norskov JK, Mavrikakis M (2002) Annu Rev Phys Chem 53:319

18. Christensen CH, Norskov JK (2008) J Chem Phys 128:182503 
19. Jones G, Bligaard T, Abild-Pedersen F, Norskov JK (2008) J Phys Condens Matter 20:064239

20. Norskov JK, Bligaard T, Hvolbaek B, Abild-Pedersen F, Chorkendorff I, Christensen CH (2008) Chem Soc Rev 37:2163

21. Nilsson A, Pettersson LGM, Nørskov JK (eds) (2008) Chemical bonding at surfaces and interfaces. Elsevier, Amsterdam

22. Besenbacher F, Chorkendorff I, Clausen BS, Hammer B, Molenbroek AM, Norskov JK, Stensgaard I (1998) Science 279:1913

23. Besenbacher F, Norskov JK (2000) Science 290:1520

24. Helveg S, Lauritsen JV, Laegsgaard E, Stensgaard I, Norskov JK, Clausen BS, Topsoe H, Besenbacher F (2000) Phys Rev Lett 84:951

25. Lopez N, Norskov JK (2002) J Am Chem Soc 124:11262

26. Sehested J, Larsen KE, Kustov AL, Frey AM, Johannessen T, Bligaard T, Andersson MP, Norskov JK, Christensen CH (2007) Top Catal 45:9

27. Studt F, Abild-Pedersen F, Bligaard T, Sorensen RZ, Christensen CH, Norskov JK (2008) Science 320:1320

28. Topsoe H, Hinnemann B, Norskov JK, Lauritsen JV, Besenbacher F, Hansen PL, Hytoft G, Egeberg RG, Knudsen KG (2005) Catal Today 107-108:12

29. de Broglie L (1924) Philos Mag 47:446

30. Davisson C, Germer LH (1927) Nature 119:558

31. Davisson C, Germer LH (1927) Phys Rev 30:705

32. Somorjai GA, Rous PJ (2008) J Phys Condens Matter 20:304210

33. Pendry JB (1994) Surf Sci 299:375

34. Woodruff DP, Delchar TA (1994) Modern techniques of surface science. Cambridge University Press, Cambridge

35. Ignatjev A, Pendry JB, Rhodin TN (1971) Phys Rev Lett 26:189

36. Capart G (1971) Surf Sci 26:429

37. Lang E, Mueller K, Heinz K, Hove MAV, Koestner RJ, Somorjai GA (1983) Surf Sci 127:347

38. Kesmodel LL, Dubois LH, Somorjai GA (1979) J Chem Phys 70:2180

39. Koestner RJ, Hove MAV, Somorjai GA (1982) Surf Sci 121:321

40. Starke U, Pendry JB, Heinz K (1996) Prog Surf Sci 52:53

41. Saldin DK, Pendry JB, Vanhove MA, Somorjai GA (1985) Phys Rev B 31:1216

42. Wander A, Held G, Hwang RQ, Blackman GS, Xu ML, Andres Pd, Hove MAV, Somorjai GA (1991) Surf Sci 249:21

43. Rous PJ, Pendry JB (1989) Surf Sci 219:355

44. Zhang XG, Hove MAV, Somorjai GA, Rous PJ, Tobin D, Gonis A, MacLaren JM, Heinz K, Michl M, Lindner H, Miiller K, Ehsasi M, Block JH (1991) Phys Rev Lett 67:1298

45. Starke U, Barbieri A, Materer N, Vanhove MA, Somorjai GA (1993) Surf Sci 286:1

46. Wander A, Vanhove MA, Somorjai GA (1991) Phys Rev Lett 67:626

47. Roberts JG, Hoffer S, Van Hove MA, Somorjai GA (1999) Surf Sci 437:75

48. Materer N, Starke U, Barbieri A, Vanhove MA, Somorjai GA, Kroes GJ, Minot C (1995) J Phys Chem 99:6267

49. Materer N, Starke U, Barbieri A, VanHove MA, Somorjai GA, Kroes GJ, Minot C (1997) Surf Sci 381:190

50. Van Hove MA (2009) Surf Sci 603:1301

51. Lundgren E, Mikkelsen A, Andersen JN, Kresse G, Schmid M, Varga P (2006) J Phys Condens Matter 18:R481

52. Gustafson J, Mikkelsen A, Borg M, Lundgren E, Kohler L, Kresse G, Schmid M, Varga P, Yuhara J, Torrelles X, Quiros C, Andersen JN (2004) Phys Rev Lett 92:126102

53. Lundgren E, Gustafson J, Resta A, Weissenrieder J, Mikkelsen A, Andersen JN, Kohler L, Kresse G, Klikovits J, Biederman A, Schmid M, Varga P (2005) J Electron Spectrosc Relat Phenom 144-147:367

54. Toyoshima I, Somorjai GA (1979) Catal Rev Sci Eng 19:105
55. Hagstrum HD (1978) In: Fiermanns L, Vennik J, Dekeyser V (eds) NATO advanced study institutes series: series B, physics, vol 32. Plenum Press, New York

56. Margaritondo G, Weaver JH (1985) In: Park RL, Lagally MG (eds) Solid state physics: surfaces, methods of experimental physics, vol 22. Academic Press, New York

57. Leckey R (1992) In: O’Conner DJ, Sexton BA, Smart RSC (eds) Surface analysis methods in materials science. Springer series of surface sciences, vol 23. Springer-Verlag, Berlin

58. Briggs D (1979) In: Brundle CR, Baker AD (eds) Electron spectroscopy: theory, techniques, applications, vol 3. Academic Press, New York

59. Moulder JF, Stickle WF, Sobol PE, Bomben KD (1992) Handbook of X-ray photoelectron spectroscopy. Perkin Elmer, Eden Prairie

60. Rodriguez JA, Goodman DW (1992) Science 257:897

61. Jacobsen KW, Norskov JK, Puska MJ (1987) Phys Rev B $35: 7423$

62. Jacobsen KW, Stoltze P, Norskov JK (1996) Surf Sci 366:394

63. Bligaard T, Norskov JK (2008) In: Nilsson A, Pettersson LGM, Norskov JK (eds) Chemical bonding at surfaces, interfaces. Elsevier Science \& Technology, Amsterdam

64. Somorjai GA, Bent BE (1985) Prog Colloid Polym Sci 70:38

65. Mavrikakis M, Hammer B, Norskov JK (1998) Phys Rev Lett $81: 2819$

66. Abild-Pedersen F, Greeley J, Studt F, Rossmeisl J, Munter TR, Moses PG, Skulason E, Bligaard T, Norskov JK (2007) Phys Rev Lett 99:016105

67. Hellman A, Honkala K, Remediakis IN, Logadottir A, Carlsson A, Dahl S, Christensen CH, Norskov JK (2009) Surf Sci 603:1731

68. Honkala K, Hellman A, Remediakis IN, Logadottir A, Carlsson A, Dahl S, Christensen CH, Norskov JK (2005) Science 307:555

69. Bronsted JN (1928) Chem Rev 5:231

70. Evans MG, Polanyi M (1938) Trans Faraday Soc 34:0011

71. Boudart M (1997) In: Ertl G, Hnozinger H, Weitkamp J (eds) Handbook of heterogeneous catalysis. Wiley-VCH, Weinheim

72. Logadottir A, Rod TH, Norskov JK, Hammer B, Dahl S, Jacobsen CJH (2001) J Catal 197:229

73. Sabatier $P$ (1919) La catalyse en chimie organique. Conference[s] faite a mm. les étudiants américains, 1.-9. E Privat, Toulouse

74. Jacobsen CJH, Dahl S, Clausen BS, Bahn S, Logadottir A, Norskov JK (2001) J Am Chem Soc 123:8404

75. Andersson MP, Bligaard T, Kustov A, Larsen KE, Greeley J, Johannessen T, Christensen CH, Norskov JK (2006) J Catal 239:501

76. Bligaard T, Andersson MP, Jacobsen KW, Skriver HL, Christensen CH, Norskov JK (2006) MRS Bull 31:986

77. Bligaard T, Johannesson GH, Ruban AV, Skriver HL, Jacobsen KW, Norskov JK (2003) Appl Phys Lett 83:4527

78. Greeley J, Norskov JK (2007) Surf Sci 601:1590

79. Greeley J, Norskov JK (2009) J Phys Chem C 113:4932

80. Zhang LN, Singh S, Tian CS, Shen YR, Wu Y, Shannon MA, Brinker CJ (2009) J Chem Phys 130:154702

81. Bratlie KM, Komvopoulos K, Somorjai GA (2008) J Phys Chem C 112:11865

82. Aliaga C, Park JY, Yamada Y, Lee HS, Tsung CK, Yang PD, Somorjai GA (2009) J Phys Chem C 113:6150

83. Zhuang X, Miranda PB, Kim D, Shen YR (1999) Phys Rev B 59:12632

84. Tao F, Grass ME, Zhang YW, Butcher DR, Renzas JR, Liu Z, Chung JY, Mun BS, Salmeron M, Somorjai GA (2008) Science 322:932

85. Zaera F (2002) Appl Catal A Gen 229:75

86. Zaera F (2002) J Phys Chem B 106:4043

87. Somorjai GA, Kliewer CJ (2009) React Kinet Catal Lett 96:191 
88. Kliewer CJ, Bieri M, Somorjai GA (2008) J Phys Chem C 112:11373

89. Bratlie KM, Montano MO, Flores LD, Paajanen M, Somorjai GA (2006) J Am Chem Soc 128:12810

90. Bratlie KM, Flores LD, Somorjai GA (2005) Surf Sci 599:93

91. Yang MC, Chou KC, Somorjai GA (2004) J Phys Chem B 108:14766
92. Loffreda D, Delbecq F, Vigne F, Sautet P (2005) Angew Chem Int Ed 44:5279

93. Loffreda D, Jugnet Y, Delbecq F, Bertolini JC, Sautet P (2004) J Phys Chem B 108:9085

94. Yoon HA, Salmeron M, Somorjai GA (1997) Surf Sci 373:300 